\title{
Ignition and Front Propagation in Polymer Electrolyte Membrane Fuel Cells
}

\author{
J. B. Benziger, E.-S. Chia, Y. De Decker, and I. G. Kevrekidis \\ Department of Chemical Engineering, Princeton University, Princeton, NJ 08544, USA
}

(Dated: September 23, 2018)

\begin{abstract}
Water produced in a Polymer Electrolyte Membrane (PEM) fuel cell enhances membrane proton conductivity; this positive feedback loop can lead to current ignition. Using a segmented anode fuel cell we study the effect of gas phase convection and membrane diffusion of water on the spatiotemporal nonlinear dynamics -localized ignition and front propagation- in the cell. Co-current gas flow causes ignition at the cell outlet, and membrane diffusion causes the front to slowly propagate to the inlet; counter-current flow causes ignition in the interior of the cell, with the fronts subsequently spreading towards both inlets. These instabilities critically affect fuel cell performance.
\end{abstract}

PACS numbers: 82.47.Gh,82.47.-a,47.54.-r

Fuel cells constitute a reliable and environmentally friendly alternative energy source. Beyond steady state operation, understanding of their startup and transient dynamic behavior is crucial to their use in variable load automotive applications [1, 2]. These transient dynamics are intensely nonlinear due to a positive feedback loop: we recently demonstrated that water generated in a polymer electrolyte membrane (PEM) fuel cell increases proton transport exponentially, which "ignites" the current 3, 4]. There is a strong analogy to the positive feedback loop leading to nonlinear dynamics such as ignition and spatiotemporal front propagation in exothermic chemical reactions. There, a product of the reaction (heat) enhances the reaction rate by raising the temperature [5]. In autohumidified PEM fuel cells, the positive feedback between membrane water activity and proton conductivity is known to cause steady state multiplicity [6].

Here we present spatiotemporal nonlinear dynamic phenomena (current ignition and current density front propagation along flow channels) in a two-dimensional fuel cell. PEM fuel cells typically have flow channels that distribute the fuel (hydrogen) across the anode and oxidizer (oxygen) across the cathode (Fig. 10 7]. Longitudinal water gradients in the membrane produce a sharp current density front that propagates in time along the channel 8]. To design and control variable load, dynamic fuel cell operation, the mechanism underlying such dynamics must be understood.

Experimental. Fig. 2] shows a photograph of a simplified fuel with a segmented anode, that permits current profile measurements. The current through each segment of the anode and the voltage drop across the external load resistor are recorded as a function of time. The cell operates at atmospheric pressure; the flow rates of hydrogen at the anode and oxygen at the cathode are maintained with mass flow controllers.

The fuel cell is placed between two temperature controlled aluminum blocks. There are six segments to the fuel cell, each with an active area of $0.5 \mathrm{~cm}^{2}$. It is preconditioned with a $20 \Omega$ load at $60{ }^{\circ} \mathrm{C}$ and flow rates of $3.5 \mathrm{sccm} \mathrm{H}_{2}$ at the anode and $\mathrm{O}_{2}$ at the cathode for 8-12

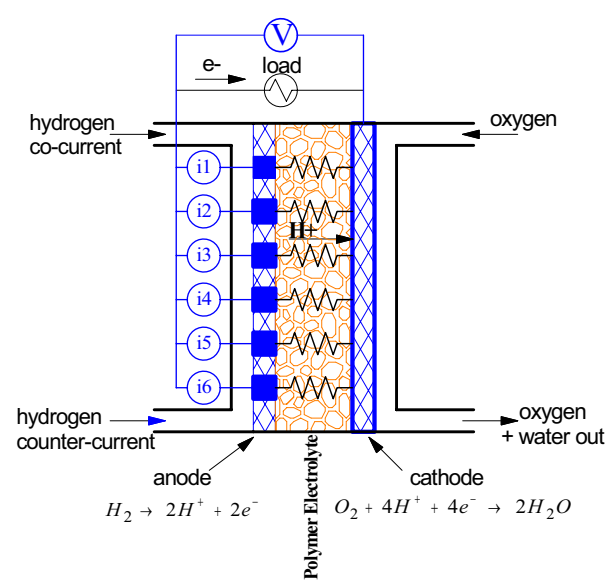

FIG. 1: (Color online) Schematic of a Hydrogen-Oxygen PEM fuel cell including internal and external equivalent circuit elements. Hydrogen molecules dissociatively adsorb at the anode and are oxidized to protons. Electrons travel through an external load resistance to the cathode, while protons diffuse through the PEM under an electrochemical gradient to the cathode. Oxygen molecules adsorb at the cathode, are reduced, and react with the protons to produce water. The product water is absorbed into the PEM, or evaporates into the gas streams at the anode and cathode.

hours. The current in each segment was $<1 \mathrm{~mA}$ after preconditioning (extinction).

The external load was then reduced to $0.25 \Omega$ keeping temperature and flow rates fixed. The current in each segment remained at $<1 \mathrm{~mA}$ for more than 4 hours, at which time $100 \mu \mathrm{L}$ of water was injected into the anode feed stream. The current response for each segment after the water injection is shown in Fig. 3. The injection of the water "ignited" the fuel cell current. Before water injection the resistance of the dry membrane for proton conduction was very high $\left(>10 \mathrm{k} \Omega \mathrm{cm}^{-2}\right)$, limiting the current and hence the water production. The injected water was absorbed into the membrane, decreasing the membrane resistance to $\sim 1 \Omega \mathrm{cm}^{-2}$; this increased 


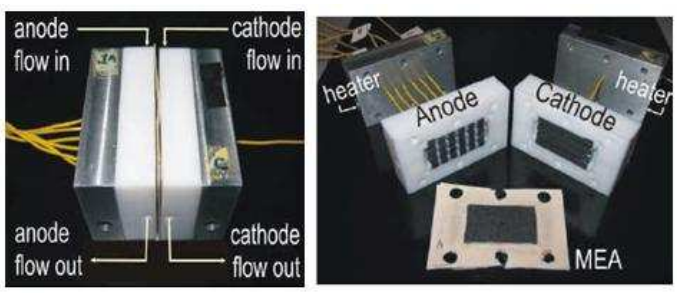

FIG. 2: (Color online) The segmented anode fuel cell. The anode electrode was broken into six individual elements separated by Teflon spacers. Current through each element was measured independently. A membrane-electrode-assembly employing 2 ETEK electrodes with carbon supported Pt catalyst and a Nafion 115 membrane was placed between anode and cathode.

the currents to $\sim 100 \mathrm{~mA}$ and made the fuel cell selfsustaining.

To explore the location and dynamics of ignition in the flow channels, the fuel cell was extinguished as described above. The temperature was then reduced to 25 ${ }^{0} \mathrm{C}$, decreasing the water removal, and the load resistance reduced to $0.25 \Omega$, increasing the water production. Current and voltage were recorded every $100 \mathrm{~s}$ for a period of 20 hours after these changes. For the measurements reported here, the fuel cell was placed with the flow channels running vertically. Co-current measurements were made with both $\mathrm{H}_{2}$ and $\mathrm{O}_{2}$ flows going from top to bottom; for counter-current measurements, the oxygen flow went from top to bottom and the hydrogen flow from bottom to top.

The ignition and subsequent current distributions along the flow channels as functions of time are shown in Figs. 目(a) and 4 (b). An induction period of hours was required before any significant current was measured; the length of this induction period depended on how dry the fuel cell membrane was before startup (i.e. before reducing the load resistance.) For co-current flow (Fig प(a)) ignition first occurred at the outlet of the fuel cell; the current in anode element 6 , near the outlet, rose from $<$ $1 \mathrm{~mA}$ to $\sim 100 \mathrm{~mA}$ over a period of $5 \mathrm{~min}$. A current ignition front then propagated from element 6 to element 1 over a period of 15-20 minutes; as the current front propagated towards the entrance of the flow channels, the current at the exits of the flow channels dropped. With counter-current flow ignition first occurred at element 3, at the interior of the flow channel (Fig. 廿(b)). From the center, the ignition fronts "fanned" outwards, but the highest current always occurred in the center of the flow channel. The induction period was also longer with counter-current flow than with co-current flow. The long-term, total steady state steady state current was roughly double for counter-current (as opposed to cocurrent) flow.

Modeling. We have been able to capture the basic

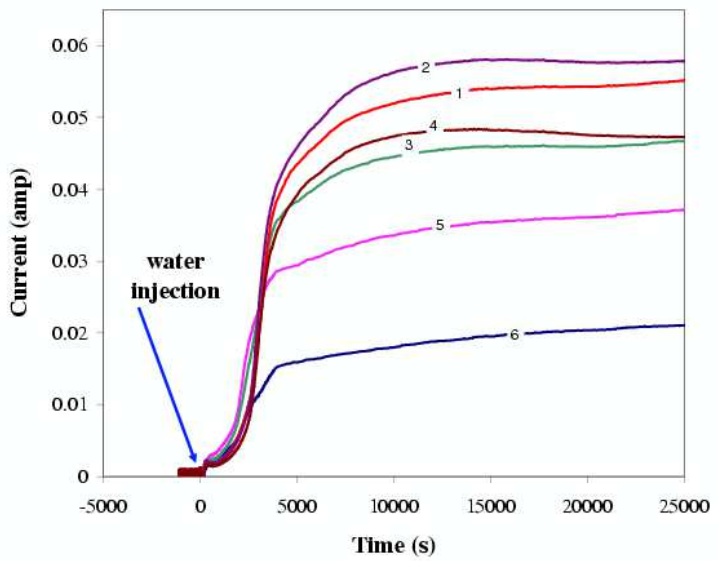

FIG. 3: Fuel cell ignition by water injection. The fuel cell was preconditioned so as to dry the membrane; the current in each segment of the anode was then $<1 \mathrm{~mA}$. At $\mathrm{t}=0,100 \mu \mathrm{L}$ of water were injected into the anode (hydrogen) feed. After the water was absorbed into the membrane, the fuel cell currents rose dramatically (ignited), and remained so. The numbers refer to the currents in the segments in order - 1 is at the anode inlet and 6 is at the anode outlet.

physics of ignition dynamics in a simple lumped differential, single anode fuel cell [6]; to capture localized ignition and spatiotemporal front propagation the model is extended to a series of differential elements. The key features of the model are the water inventory in the polymer electrolyte, the transverse proton conductivity from the anode to the cathode and the longitudinal water transport through the membrane. Water sorbed into the polymer ionizes sulfonic acid groups, facilitating proton transport; water sorption by the PEM is limited by the total number of sulfonic acid groups in the membrane. Water is sorbed into, or desorbed from, the membrane depending on the balance between water evaporating into the gas flow channels and water produced by the fuel cell. The multilayered membrane-electrode-assembly in PEM fuel cells results in complex intra- and interlayer transport processes. Since the flow channels are much longer than the membrane thickness $(5 \mathrm{~cm}$ vs. $0.0125 \mathrm{~cm}$ ), we make the simplifying assumption that water activity in the membrane is in local equilibrium with water activity in the gas flow channel above it, and the only gradients are longitudinal along the channel.

The water balance in each differential element $j$ of the membrane is given by eq. 1 for co-current gas flow $(j=1$ to $6 ; j=0$ and $j=7$ denote the anode inlet and outlet, respectively; the counter-current case easily follows); the 

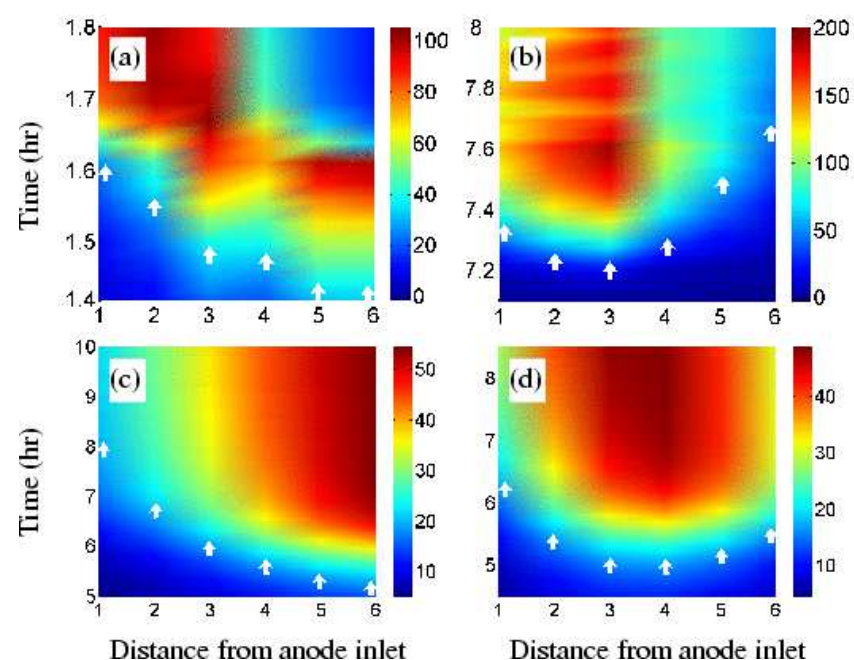

FIG. 4: (Color online) A comparison of the experimental and computed currents for co-current and counter-current flow of hydrogen and oxygen in a segmented anode PEM fuel cell. The color scale is for current through each anode segment in mA. (a) experimental co-current (b) experimental counter-current (c) computed co-current (d) computed counter-current. For the simulations, the flow rate for $\mathrm{H}_{2}$ and $\mathrm{O}_{2}$ is 3.5 and $6.5 \mathrm{~mL} \mathrm{~min}{ }^{-1}$ respectively and $k_{m}=3 \times 10^{-6}$ $\mathrm{mol} \mathrm{s}^{-1}$.

inventory is balanced by water produced $(1 / 2$ the proton current), water convected in the gas flow, and longitudinal water diffusion (described by a lumped mass transfer coefficient between differential elements). Eq. 2 is an empirical fit to the number of water molecules associated with each sulfonic acid group, $\lambda$, as a function of water activity $\alpha_{w}$ in a Nafion 115 membrane [9].

$$
\begin{aligned}
& {\left[N_{\mathrm{SO}_{3}} \frac{d \lambda(j)}{d a_{w}(j)}+\left(V_{A}+V_{C}\right) \frac{P_{w}^{0}}{R T}\right] \dot{a}_{w}(j)=} \\
& \frac{i(j)}{2 \mathcal{F}}+\left[F_{A}(j-1)+F_{C}(j-1)\right] P_{w}(j-1) \\
& -\left[F_{A}(j)+F_{C}(j)\right] P_{w}(j)+k_{m}[a(j+1)+a(j-1) \\
& -2 a(j)] \\
& \quad \begin{aligned}
\lambda(j)= & 14.9 a_{w}(j)-44.7 a_{w}^{2}(j)+70 a_{w}^{3}(j) \\
& -26.5 a_{w}^{4}(j)-0.446 a_{w}^{5}(j)
\end{aligned}
\end{aligned}
$$

We assume that the total gas pressure $P_{T}$ is fixed, and the local water activity in the membrane is in equilibrium with the local water partial pressure $P_{w}$, i.e. $P_{w}(j)=$ $a_{w}(j) P_{w}^{0}$ and $P_{\mathrm{H}_{2}}(j)=P_{\mathrm{O}_{2}}(j)=P_{T}-P_{w}(j) ; P_{w}^{0}$ is the water vapor pressure at this temperature. The molar flow rates change along the flow channel as water is formed; the molar flows are given by $F_{A}(j)=F_{A}(j-1)-i(j) / 4 \mathcal{F}$ and $F_{C}(j)=F_{C}(j-1)$; the subscript $A(C)$ corresponds to the anode(cathode), $i(j)$ is the local current and $\mathcal{F}$ the Faraday constant. Lastly, we assume that the local potential $V_{F C}$ between the anode and cathode is dictated by the thermodynamic driving force as in eq. 3 This neglects interfacial potential drops, which results in the predicted currents being about $20 \%$ larger than found in real fuel cells.

$$
V_{F C}(j)=1.23-\frac{R T}{4 \mathcal{F}} \ln \left[\frac{P_{\mathrm{H}_{2}}^{2}(j) P_{\mathrm{O}_{2}}(j)}{P_{\mathrm{atm}}^{3} a_{w}^{2}(j)}\right]
$$

Based on the equivalent electrical circuit, the differential elements are electrically connected in parallel to each other. The voltage across the external load resistance thus depends on the total current produced by all elements; the local current is given by eq. 4. The local membrane resistance, $R_{M}(j)$, depends of the local water content in the membrane. For a Nafion 115 membrane employed in this fuel cell the membrane resistance as a function of water activity is given by eq. [5] [9].

$$
\begin{aligned}
& i(j)=\frac{V_{F C}(j)-R_{L} \sum_{k \neq j} i(k)}{R_{M}(j)+R_{L}} \\
& R_{M}(j)=5 \times 10^{5} \exp \left(-14 a_{w}^{0.2}\right)
\end{aligned}
$$

In a single, differential PEM fuel cell ignition occurs when the initial water content in the membrane is sufficient for water production to exceed water removal by convection. Here ignition will occur when (and where!) the water production exceeds water removal, i.e. when (and where) the right hand side of eq. प becomes greater than zero. Water production depends on the load resistance and the membrane resistance. A dry membrane has a resistance of $500 \mathrm{k} \Omega \mathrm{cm}^{-2}$, limiting the current density to a maximum of $2.4 \mu \mathrm{A} \mathrm{cm}^{-2}$. According to eq. 1 the feed flow rates would have to be $<0.1 \mathrm{~mL} \mathrm{hr}^{-1}$ at a fuel cell temperature of $60{ }^{0} \mathrm{C}$ for water production to be greater than water removal and ignite the fuel cell. Absorption of $10 \mu \mathrm{L} \mathrm{cm}^{-2}$ of water into the membrane reduces its resistance to $\sim 10 \Omega \mathrm{cm}^{-2}$ and the maximum current density is $100 \mathrm{~mA} \mathrm{~cm}{ }^{-2}$, sufficient for water production to exceed water removal, and the fuel cell to ignite as shown in Fig. 3

The key elements that account for ignition are (1) an exponential dependence of proton conductivity in the PEM with membrane water content and (2) the dynamics of water uptake into the PEM. The location of ignition and front propagation are consequences of (1) convection of water produced towards the cell downstream, where it can accumulate and (2) diffusion of water upstream through the polymer membrane. Figs. 近 (c) and (d) show the simulated current profiles for co-current and counter-current flow with $T=47^{0} \mathrm{C}, R_{L}=5 \Omega$. The model captures both the ignition and the front propagation, though it overpredicts the experimentally observed currents.

The model is only semi-quantitative because it neglects finite water mass transfer rates into the membrane 
and from the membrane into the gas phases. It also neglects the effects of condensing liquid water, hindering gas transport from the flow channels to the membrane/electrode interface. More detailed models that incorporate these effects can give quantitative fits to experimental results; yet the added complexity does not significantly enhance physical understanding.

For co-current flow the water produced upstream is conducted towards the outlet, where it slowly accumulates in the membrane; when the water content increases to the point where the local membrane resistance becomes comparable to the external load resistance the current starts increasing rapidly, hydrating the membrane and causing ignition at the outlet of the flow channel. Upon ignition the water activity in the membrane approaches unity. Water is then transported upstream through diffusion in the membrane itself, causing upstream propagation of the ignition. The model did not capture the observed eventual decrease in the downstream current, after the ignition propagated to the inlet of the fuel cell. This decrease is due to condensing water accumulating in the cathode, inhibiting oxygen transfer to the catalyst and reducing the current.

In counter-current flow, water formed at the cathode is convected towards the anode inlet in the cathode flow channel. It is also transported across the membrane to the anode, where it is convected towards the anode outlet. Water accumulates fastest towards the middle of the flow channels, resulting in an interior ignition point. The ratio of the flow rates between the anode and cathode affects ignition point location; a relative increase of the anode flow rate shifts ignition towards the anode outlet. After ignition, water starts to accumulate locally in the membrane. The transport of water through the membrane from high concentration at the middle of the flow channel towards the outlets results in the "fanning out" of the ignition fronts.

Liquid water was observed leaving the flow channels 30-40 minutes after ignition. Ignition takes the fuel cell from very low water activity to water activity of unity: condensation of liquid water in the flow channels. Gravity plays a key role in how such liquid water moves through the flow channels; the operation changes dramatically if gas flow in the channel is counter to gravity driven liquid water flow. When the fuel cell was vertical, gravity caused the liquid to drain, and permitted good access for the reactants from the flow channels to the electrode/electrolyte interface. When the fuel cell was horizontal the initial ignition phenomena were similar to those reported in Fig. 3. after ignition, however, large fluctuations in the local current density appear to correlate with water droplets exiting the cell. In the horizontal orientation, liquid water condensing in the flow channels could partially block flow. The liquid drops were pushed along the flow channels by the flowing gas, but in an irregular fashion, that gave rise to large fluctuations in the local current density. Transport of liquid water in the flow channels is not accounted for at our level of modeling. The model can thus capture ignition and front propagation, but will break down at longer times, when liquid water floods the cathode gas diffusion layer (GDL) and starts entering the flow channels.

We have demonstrated how the water exponentially increases proton conductivity in polymer electrolyte membranes leading to ignition of the current in PEM fuel cells. Water can be injected to a fuel cell to ignite the current, just like a match can be struck to ignite a flame. Transport of water laterally, coupled with the exponential increase in proton conductivity produces current fronts that propagate along flow channels, just like flame fronts. The positive feedback loop between water production and increased proton conductivity of the electrolyte membrane in PEM fuel cells is analogous to exothermic chemical reaction ignition and flame propagation, yet "water fans the flame" in PEM fuel cells! Front propagation depends on flow configurations creating different front ignition and propagation patterns. Understanding the parametric dependence and time scales of these phenomena is a vital component of fuel cell design, non-steady state operation and control.

Acknowledgements We thank the National Science Foundation (CTS -0354279 and DMR-0213707) for support of this work. E. Chia thanks the Princeton University Program in Plasma Science and Technology, and U.S. Department of Energy Contract No. DE-AC02-76-CHO3073 for fellowship support. Y. De Decker thanks the Belgian American Educational Foundation for financial support.

[1] A. Martin in What we have learned from vehicle demonstration programs?, Ninth Grove Fuel Cell symposium, London, UK, 4-6 October, 2005; Elsevier: London, UK.

[2] M. Fronk in Fuel Cell Vehicle Commercialisation, Ninth Grove Fuel Cell Symposium, London, UK, 4-6 October, 2005; Elsevier: London, UK.

[3] J. M. Moxley, S. Tulyani, and J. B. Benziger, Chem. Eng. Sci. 58, 4705 (2003).

[4] E. J. Chia, J. B. Benziger, and I. G. Kevrekidis, AIChE J. 50, 2320 (2004).

[5] D. Luss, D. Ind. Eng. Chem. Res. 36, 2931 (1997); B. Marwaha and D. Luss, Chem. Eng. Sci. 58733 (2003); B. Marwaha, S. Sundarram and D. Luss, Chem. Eng. Sci. $\mathbf{5 9 ,} 5569$ (2004).

[6] J. B. Benziger et al., AIChE J. 50, 1889 (2004); J. B. Benziger et al., Chem. Eng. Sci. 60, 1743 (2005).

[7] P. Berg, K. Promislow et al., J. Elec., Soc. 151, A341 (2004).

[8] S. Dutta et al., J. Appl. Elec. 30, 135 (2000); W. K. Lee et al., J. Elec. Soc. 151 A341 (2003).

[9] C. Yang et al., J. Membr. Sci. 237, 145 (2004). 